\title{
Relaciones entre la precipitación, producción de biomasa e índices espectrales de la vegetación: alcances y limitaciones
}

\author{
Relationships among precipitation, biomass production and spectral \\ vegetation indices: scopes and limitations
}

\author{
Fernando Paz Pellat ${ }^{1 *}$ y Heriberto Díaz Solís ${ }^{2}$
}

\footnotetext{
${ }^{1}$ GRENASER. Colegio de Postgraduados. Carretera México-Texcoco, km 36.5. 56230 Montecillo, Estado de México, México.

*Autor responsable (ferpazpel@gmail.com)

${ }^{2}$ Departamento de Recursos Naturales, Universidad Autónoma Agraria Antonio Narro. Calzada Antonio Narro 1923, Buenavista. 25315 Saltillo, Coahuila, México.
}

\section{RESUMEN}

La precipitación (P) es uno de los factores principales que explican la producción de biomasa en pastizales y matorrales. La eficiencia en el uso de la precipitación (EUP), relación entre la producción de biomasa y la precipitación, permite el uso de paradigmas simples de modelos (Simple Ecological Sustainability Simulator o SESS) que pueden ser parametrizados con información meteorológica existente en el país. La relación EUPPrecipitación se analizó de acuerdo con la evidencia experimental disponible, estableciéndose la relación matemática congruente con esta evidencia, a efecto de replantear la relación que se usó en el modelo SESS. Para parametrizar la EUP en función de índices de vegetación, se analizó un ejemplo en Coahuila, México, de corridas del modelo SESS y de estimaciones del índice NVDIcp a través del uso de imágenes del sensor AVHRR, para establecer relaciones funcionales entre la EUP espectral (NDVIcp/P) y la biomasa verde estimada con el modelo SESS. Al final se discuten opciones de desarrollo para enfoques de integración de modelos y sensores remotos.

Palabras clave: eficiencia en el uso de la precipitación, NDVIcp, SESS, relaciones funcionales.

\section{SUMMARY}

Precipitation is one of the main factors that explain production of biomass in grassland and shrubland.
Precipitation use efficiency (PUE), the ratio between biomass production and precipitation, allows the use of simple modeling paradigms (Simple Ecological Sustainability Simulator or SESS) that can be parameterized with weather information existing in the country. The PUE-Precipitation relationship is analyzed based on available experimental evidence, establishing a consistent mathematical relationship congruent with this evidence to reconsider the ratio used in the SESS model. To parameterize the PUE in function of vegetation indexes (IV), an example in Coahuila, Mexico, is analyzed of SESS model runs and NVDIcp index estimates using images from AVHRR sensor, to establish functional relationships between spectral PUE (NDVIcp/P) and the green biomass estimated with SESS model. Finally, development options for approaches to integrating models and remote sensing are discussed.

Index words: rainfall use efficiency, NDVIcp, SESS, functional relations.

\section{INTRODUCCIÓN}

El desarrollo de seguros ganaderos paramétricos satelitales (seguros con base en parámetros o índices) requiere que su riesgo base sea mínimo. El riesgo base está asociado a las diferencias "en campo" con relación a la vegetación observada o medida y las estimadas usando índices o indicadores. Así, si los índices utilizados para modelar la respuesta de
Cita recomendada:

Paz Pellat, F. y H. Díaz Solís. 2018. Relaciones entre la precipitación, producción de biomasa e índices espectrales de la vegetación: alcances y limitaciones. Terra Latinoamericana 36: 153-168.

DOI: https://doi.org/10.28940/terra.v36i2.235
Recibido: abril de 2017. Aceptado: marzo de 2018 . Terra Latinoamericana 36: 153-168. 
la vegetación ante el clima y manejo, principalmente, tienen fundamentos biofísicos robustos, entonces los productos del seguro tendrán bases sólidas y no sujetos factores de riesgo adicionales.

La tecnología de los sensores remotos ha sido utilizada para el desarrollo de índices de la vegetación (IV) espectrales que están relacionados con la biomasa aérea $(\mathrm{Bm})$ e índice de área foliar (IAF) (AGROASEMEX, 2006; Paz et al., 2006). Aunque el diseño de los seguros paramétricos fue elaborado para obtener una relación del tipo $\mathrm{Bm}=a \mathrm{IV}$, donde $a$ es una constante empírica (AGROASEMEX, 2006), su uso cuantitativo (producción forrajera) está limitado por falta de conocimiento de las constantes $a$ en términos espaciales. Una alternativa explorada en este trabajo es la relación de los patrones temporales de los IV (crecimiento y desarrollo de la vegetación) con modelos que tengan una base biofísica y que sean parametrizados en forma simple. Así, se usó un concepto de asimilación, donde los patrones de los IV pueden alimentar a los modelos biofísicos para lograr una integración de los esquemas de simulación (modelos) con la de la tecnología satelital (mediciones indirectas).

Los modelos biofísicos (con base en procesos biológicos y su parametrización) presentan problemas con relación al principio de parsimonia, ya que requieren de una gran cantidad de datos de entrada, los cuales deben ser espacialmente explícitos para que se puedan utilizar en conjunto con los IV. Los modelos biofísicos complejos modelan la relación atmósferavegetación-suelo por compartimentos y usan pasos temporales con diferentes resoluciones; aunque para las aplicaciones ganaderas hay modelos relativamente complejos o subcomponentes de estos (Wight and Skiles, 1987; Arnold et al., 1995; Donnelly et al., 1997; Freer et al., 1997; Stuth et al., 2003), su uso está restringido a sitios experimentales y resultan poco útiles en términos operacionales.

Un modelo simple que se puede acoplar con los IV es el modelo SESS (Simple Ecological Sustainability Simulator) desarrollado por Díaz-Solís et al. (2003). Así, en este trabajo se exploran los alcances y limitaciones de este modelo y se analiza una aproximación para su parametrización. Al final se discuten líneas de desarrollo para la expansión de la aproximación usada en forma más generalizada.

\section{MATERIALES Y MÉTODOS}

\section{Eficiencia en el Uso de la Precipitación}

La relación entre la precipitación $(\mathrm{P})$, total $\mathrm{o}$ promedio, y la biomasa aérea anual Bm (equivalente a la producción primaria neta anual; particularmente al considerar ciclos de crecimiento anuales como en los pastizales) ha sido discutida en muchos trabajos (Lieth, 1975; Le Houerou y Hoste, 1977; Lauenroth, 1979; Webb et al., 1983), que muestran una relación razonablemente lineal entre ambas variables, dependiente de la escala. Así, Le Houerou (1984) propuso el concepto de Eficiencia en el Uso de la precipitación (EUP) como un marco integrador del clima y la respuesta de la vegetación:

$$
E U P=\frac{B m}{P}
$$

Además de la precipitación, la evapotranspiración (ET) muestra una fuerte correlación con la $\mathrm{Bm}$ (Rosenzweig, 1968; Webb et al., 1983; Gómez y Gallopín, 1991). Otros autores muestran que la temperatura también tiene una correlación importante con la Bm (Epstein et al., 1997; Christie, 1981; Schuur, 2003); aunque Gómez y Gallopín (1991) argumentan que solo las relaciones con P y ET son significativas. En ecosistemas de bosques/selvas, la temperatura (máxima o promedio) y la radiación solar muestran relaciones significativas con la Bm (Webb et al., 1983; Huxman et al., 2004).

Para poner en contexto la relación (1) de la EUP, se puede usar el enfoque que se ha usado en modelos biofísicos relativamente complejos (Goudriaan y Van Laar, 1994; Arnold et al., 1995; Stockle et al., 2003):

$$
\begin{aligned}
& B m=\sum_{i=1}^{n} d B m_{i}=\sum_{i=1}^{n}\left(d B m p_{i}\right)\left(R C_{i}\right) \\
& d B m=(d B m p)(R C) \\
& d B m p=(e)\left(f_{R F A}\right)(R F A) \\
& R C=\operatorname{Min}\left(\text { agua, nutrientes, temp, DPV }, C_{2}, \text { etc. }\right)
\end{aligned}
$$

donde: $\mathrm{d}$ se refiere a pasos discretos $(\mathrm{i}=1,2, \ldots, \mathrm{n})$ de la acumulación de la Bm; dBmp es el incremento potencial debido solo a la actividad fotosintética de 
conversión de la radiación solar; $\mathrm{RC}$ es un factor de restricción al crecimiento (estrés hídrico, nutrimental, de temperatura, de déficit de presión de vapor - DPV, de $\mathrm{CO}_{2}$, etc.); e es la eficiencia de conversión de radiación fotosintética a biomasa (fotosintatos); $\mathrm{f}_{\mathrm{RFA}}$ es la fracción absorbida de la radiación fotosintética activa (RFA); y Min es el operador de mínimos.

La definición de la EUP implica una relación lineal entre la Bm y la P:

$B m=E U P x P$

Al comparar la relación (3) con la (2) es claro que la EUP es un factor integrador de varias variables que se puede argumentar como razonable cuando la restricción del crecimiento más importante es el agua (Huxman et al., 2004).

Dada la relación inversa entre P y ET (Le Houerou y Hoste, 1977), la relación (3) es semejante en concepto a la usada por FAO (Doorenbos y Kassam, 1979):

$\left(1-\frac{Y a}{Y p}\right)=K y\left(1-\frac{E T a}{E T p}\right)$

donde: Y es el rendimiento de un cultivo (biomasa acumulada multiplicada por el índice de cosecha) y a se refiere a la condición actual y $p$ a la potencial (sin estrés) y Ky es un factor (integrador) de proporcionalidad.

La ventaja de la relación (4) es que está formulada en función de una referencia potencial, lo que permite construir índices relativos asociados a diferentes niveles de estrés. Algo similar se puede hacer para el caso de la EUP al modificarla por efecto de estrés hídrico (cantidad de precipitación, Bai et al., 2008).

En los análisis empíricos, hasta cierto nivel de $\mathrm{P}$ (alrededor de $300 \mathrm{~mm}$ ) y con predominio en pastizales, se obtienen relaciones del tipo (Sala et al., 1988; Yahdjian y Sala, 2006):

$$
B m=-a+b P
$$

Aunque Verón et al. (2005) argumentaron que $b$ en la relación (4) no es la EUP, estos autores propusieron el uso de una precipitación efectiva (asociada a $\mathrm{Bm}=0$ ), de tal forma que la EUP se puede obtener a partir de:

$$
B m=b\left[P-\frac{a}{b}\right]=b P e
$$

donde: Pe es la precipitación efectiva. Sala et al. (1988) y Bai et al. (2008) usaron este esquema para comparar las EUP de diferentes ecosistemas y estadios seriales de éstos. No obstante, lo anterior, algunos autores (Huxman et al., 2004; Yahdjian y Sala, 2006, entre otros) muestran una constante $a$ positiva. En razón de los argumentos, ambas situaciones son factibles en términos biofísicos. Por un lado, aun cuando la precipitación fuera nula en un año, es posible obtener un crecimiento positivo (fuente de agua subterránea), aunque muy reducido. El caso contrario muestra que se requiere un mínimo de precipitación para obtener un crecimiento positivo. En lo general, por diversos argumentos más o menos generales, uno esperaría que $\mathrm{a} \approx 0$.

En la discusión anterior se han utilizado ciclos anuales del crecimiento para los análisis mostrados. Esta consideración será mantenida en lo siguiente, por lo que debe tenerse cuidado al interpretar resultados de relaciones entre la $\mathrm{Bm}$ y $\mathrm{P}$ en términos de promedios.

La variación de la pendiente $b$ (EUP) depende de los niveles de precipitación (Figura 1), donde se incrementa en años buenos (alta precipitación) y disminuye en los malos (Sala et al., 1988; Huxman et al., 2004; Bai et al., 2008). Los cambios en la pendiente $b$ han sido interpretados como producto de la condición del agostadero (Varnamkhasti et al., 1995; Le Houerou,

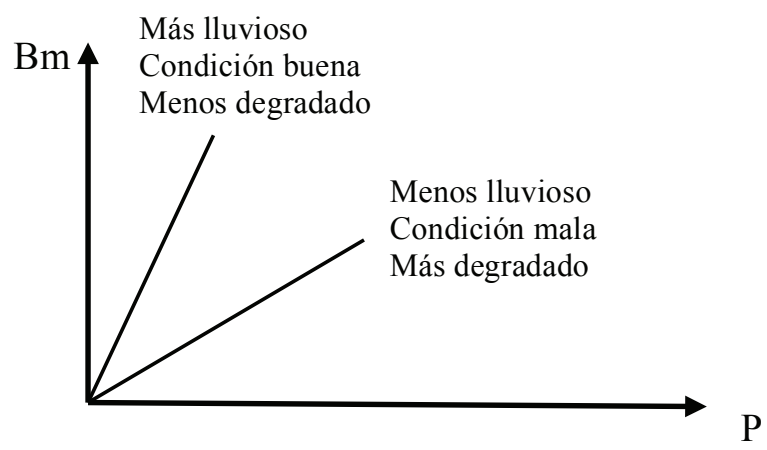

Figura 1. Efecto del nivel de precipitación/condición del agostadero/degradación en la eficiencia en el uso de la precipitación (EUP). 
1989 y 2006; Guevara et al., 2009) o de la degradación del terreno (Prince et al., 1998). La condición del agostadero implica el concepto de degradación (perdida de la capacidad productiva) y viceversa, por lo que puede definirse a través del uso de metodologías de COTECOCA (1967) o relativamente actuales (NRCS, 1997). Estas metodologías estiman el efecto de cambios en la producción forrajera producto de diferentes condiciones alejadas de una referencia (estado final único o múltiple), por lo que implícitamente resultan adecuadas para analizar los efectos de la degradación/ condición del agostadero en la EUP.

Es importante señalar que el modelo conceptual mostrado en la Figura 1 es para niveles bajos de la precipitación (menores que 300-400 $\mathrm{mm}$ ) y parte de un intercepto nulo $(a=0)$. En la Figura 2 se muestran las estimaciones para una región de Argentina para diferentes condiciones del agostadero (Blanco et al., 2005).

Las líneas P-Bm de la Figura 2 muestran cambios simultáneos en la pendiente (mayor a medida que el nivel de la condición del agostadero aumenta) e intercepto (menores al disminuir la condición del agostadero). Si observamos los patrones de las líneas P-Bm de la Figura 2, al incrementarse P arriba de 300 $\mathrm{mm}$ los efectos de la precipitación en la producción de Bm se revierten. Este ejemplo pone en perspectiva el uso de regresiones estadísticas sin un modelo conceptual suficientemente robusto para interpretar las observaciones biofísicas.

Con relación a las condiciones edáficas de un sitio, Noy-Meir (1973) planteo la hipótesis de la textura inversa, donde argumenta que la producción en regiones secas es mayor en suelos de textura gruesa (arenosos) que los de textura fina (arcillosos); es decir, a menor

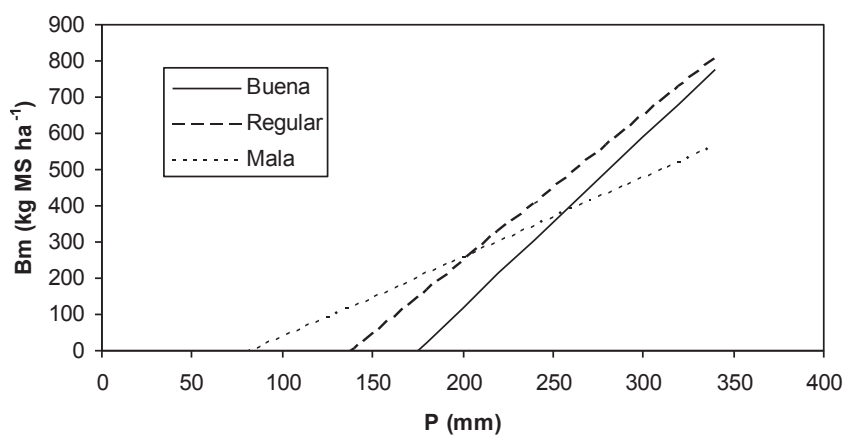

Figura 2. Relaciones (P) precipitación - (Bm) biomasa (producción) para diferentes condiciones del agostadero en una región de Argentina. capacidad de retención de humedad del suelo, mayor producción. Sala et al. (1988) muestran evidencia que sustenta esta hipótesis, pero hasta cierto umbral de P (300-370 mm). Yahdjian y Sala (2006) también ratifican la hipótesis de la textura inversa. Por otro lado, Gómez y Gallopín (1991) no encontraron que el efecto del suelo (hipótesis de la textura inversa) fuera significativo en la relación entre Bm y P o ET.

Lauenroth y Sala (1992) plantearon la hipótesis, ligada a la de la textura inversa, de que en condiciones secas (precipitación baja, condición del agostadero pobre, degradación alta), la recuperación de la EUP es menor aun cuando al año siguiente se presenten condiciones más húmedas, sugiriendo un control biótico/abiótico (vegetación y biogeoquímica) de la EUP. La evidencia experimental de Yahdjian y Sala (2006) valida esta hipótesis, donde la recuperación en la producción de $\mathrm{Bm}$ es dependiente de la intensidad de la precipitación del año anterior. Esta condición implica que la relación entre $\mathrm{Bm}$ y $\mathrm{P}$ del tiempo $\mathrm{t}$ dependen de las condiciones en el tiempo t-1. Diversos autores (Lauenroth y Sala, 1992; Yahdjian y Sala, 2006; Bai et al., 2008) han considerado esta situación en los análisis entre $\mathrm{P}$ y $\mathrm{Bm}$ con más o menos éxito. Esta situación es diferente para el caso de bosques/selvas donde la $\mathrm{Bm}$ en el tiempo t se vuelve poco relacionada con $\mathrm{P}$ en el tiempo $\mathrm{t}$ y responde mejor a la $\mathrm{Bm}$ en el tiempo t-1 y la temperatura (Huxman et al., 2004).

La evidencia experimental consignada por diversos autores (Gómez y Gallopín, 1991; Varnamkhasti et al., 1995; Knapp et al., 2008b; Guevara et al., 2009) plantea que la EUP de los pastizales y matorrales es diferente, por lo que ésta es dependiente del tipo de ecosistema, en una base global y clima similar (la Figura 3 presenta esta situación).

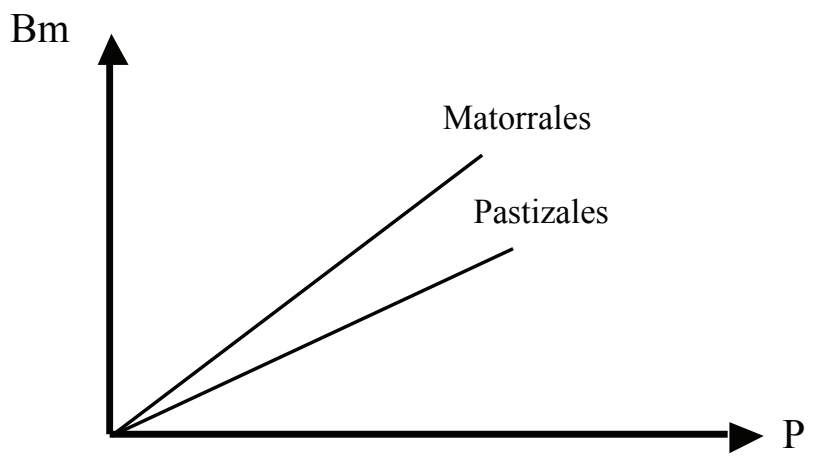

Figura 3. Eficiencia del uso de la precipitación (EUP) para pastizales y matorrales. $\mathrm{P}=$ precipitación; $\mathbf{B m}=$ biomasa. 
Los datos de Knapp et al. (2008b) plantean que los pastizales invadidos por matorrales tienen un incremento de alrededor de $400 \%$ en la producción, para un mismo régimen de precipitación. Esto implica, en la parte aérea, que la capacidad de secuestro de carbono, por ejemplo, en pastizales degradados (invadidos por matorrales) puede incrementarse substancialmente después de condiciones de degradación (sobre pastoreo o sequías extremas).

Huxman et al. (2004) plantean que para condiciones secas (EUP mínimas, Figura 2), las EUP de pastizales, matorrales y bosques/selvas son similares, lo que implica eficiencias semejantes en la conversión de precipitación a biomasa. Austin (2002) también muestran este tipo de relación (sitios más secos).

Recapitulando, hasta este punto se ha supuesto que la relación entre $\mathrm{P}$ y $\mathrm{Bm}$ es lineal (relación 4), aunque la evidencia experimental de varios autores (Gómez y Gallopín, 1991; Austin, 2002; Schuur, 2003; Huxman et al., 2004; Knapp et al., 2008a) muestran patrones no lineales con un límite superior en la respuesta de esta relación (la Bm varia muy poco con relación a incrementos de $\mathrm{P}$ después de un umbral). En condiciones muy secas, las relaciones no lineales con saturación tienen incluidas un patrón lineal (Austin, 2002; Huxman et al., 2004). En bosques/selvas y otros ecosistemas, cuando la precipitación se incrementa se presentan situaciones donde la relación P-Bm es inversamente proporcional, lineal y no lineal, sin tendencia a reducirse (Schuur, 2003; Fang et al., 2005), rompiendo el patrón de saturación (asíntota horizontal).

Un aspecto importante relacionado con el uso operativo de la EUP es el de su variación temporal y espacial. Knapp y Smith (2001) plantean que la variabilidad temporal (coeficiente de variación) es mayor en pastizales que en los matorrales. Yahdjian y Sala (2006) y Bai et al. (2008), entre otros, muestran evidencia a favor de esta hipótesis, planteando que la baja profundidad radical de los pastos los hace más vulnerables a los cambios en los niveles de precipitación; a diferencia de los matorrales (y bosques/selvas), que tienen un mayor volumen de exploración radical (agua subterránea) que les permite amortiguar los efectos de bajas precipitaciones.

En el caso de las variaciones espaciales de la EUP para un mismo ecosistema, Lauenrothh y Sala (1992), Paruelo et al. (1999) y Bai et al. (2008), entre otros, demostraron que la variabilidad espacial de la EUP es mayor que la temporal. Esto se puede interpretar como consecuencia de diferencias en la condición del agostadero/degradación en términos espaciales.

En la perspectiva de la saturación de la relación P-Bm, la dependencia de la EUP en los ecosistemas y de las variaciones espaciales, la Figura 4 muestra un esquema conceptual (linealizado) integral de estos patrones.

El modelo de la Figura 4 muestra que el caso de los patrones lineales (aproximados) para condiciones muy secas es un caso particular (no se presenta la saturación).

La discusión previa ha utilizado a la precipitación total (o promedio) en el año (o ciclo de crecimiento de la vegetación) para caracterizar la relación entre $\mathrm{P}$ y $\mathrm{Bm}$. La distribución estacional de la precipitación durante el año no ha sido considerada, que de acuerdo con el planteamiento de Le Houerou et al. (1988) estos patrones no tienen efectos significativos en la EUP. Esta hipótesis es apoyada por esquemas de modelación complejos, espacialmente explícitos, para vegetación dispersa/fragmentada (condiciones xéricas) (Kletter et al., 2009), donde la relación entre $\mathrm{P}$ y Bm presenta patrones no lineales que se pueden aproximar con funciones potenciales. La relación entre la $\mathrm{P}$ (acumulada y variación estacional) y Bm puede usarse para analizar los efectos del cambio climático en los ecosistemas terrestres (Weltzin et al., 2003; Knapp et al., 2008a).

En algunos casos la información de la Bm puede inferirse de la biomasa total en el pico del crecimiento anual de la vegetación, ya que esta correlacionada con la producción primaria neta anual o PPNA (Webb et al., 1983). Así, los patrones entre P y PPNA resultan similares (Webb et al., 1983; George et al., 1989; Wylie et al., 1992) a los discutidos previamente.

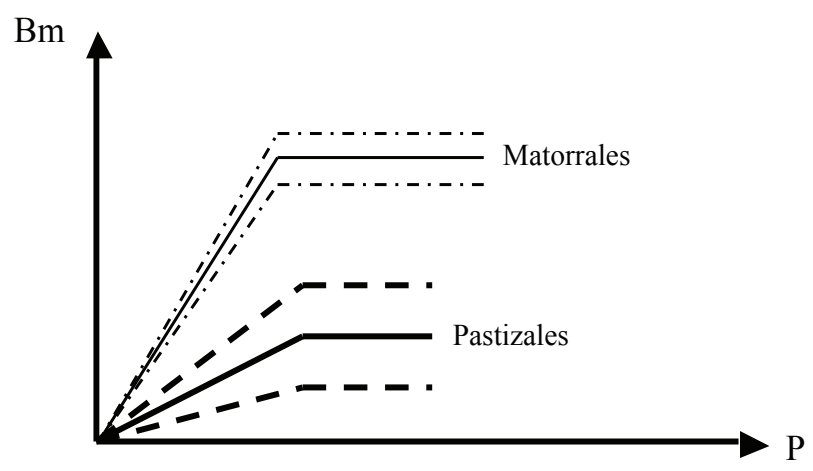

Figura 4. Modelo conceptual (linealizado) para la variación temporal-espacial de la EUP de diferentes ecosistemas. $\mathrm{Bm}=$ biomasa; $\mathbf{P}=$ precipitación). 


\section{Formas Funcionales de la Relación P-PPNA}

Con fines ilustrativos, se estimaron algunos datos (5) de la relación propuesta por Huxman et al. (2004) de la relación entre la P y PPNA (Bm), para analizar las formas funcionales que pudieran aproximar estos patrones.

La Figura 5 muestra el ajuste de varias formas funcionales para la relación P-PPNA:

$$
\begin{aligned}
& P P N A=a+b P \\
& P P N A=a+b P^{2} \\
& P P N A=a P-b P^{2} \\
& P P N A=a P^{b}
\end{aligned}
$$

donde: $a$ y $b$ son constantes empíricas. Las relaciones (6) muestran los signos usados en las constantes empíricas.

La Figura 6 muestra la relación entre las EUP y P, que es el diferencial de las relaciones (6). La ventaja de usar la relación EUP-P es que reduce las variaciones de los modelos utilizados, tal como se discute en el apartado siguiente.

En el caso del modelo lineal $(\mathrm{Y}=\mathrm{a}+\mathrm{bX}) \mathrm{y}$ polinomial de segundo grado - positivo $(\mathrm{Y}=\mathrm{aX}+$ $\mathrm{bX}^{2}$ ) solo se usaron los tres primeros datos muestrales para el ajuste, ya que el patrón general mostrado en la Figura 5 es incompatible, en términos globales, con este modelo. El modelo polinomial de segundo grado negativo $\left(\mathrm{Y}=\mathrm{aX}-\mathrm{bX} \mathrm{X}^{2}\right)$ se ajusta bien al patrón global (Figura 5).

Aunque es posible ajustar estadísticamente muchos modelos a la relación P-PPNA, solo se consideraron los que tuvieran precedentes de uso en la literatura o fueran consistentes con las observaciones experimentales. En la Figura 7 se muestra el mejor ajuste de los modelos

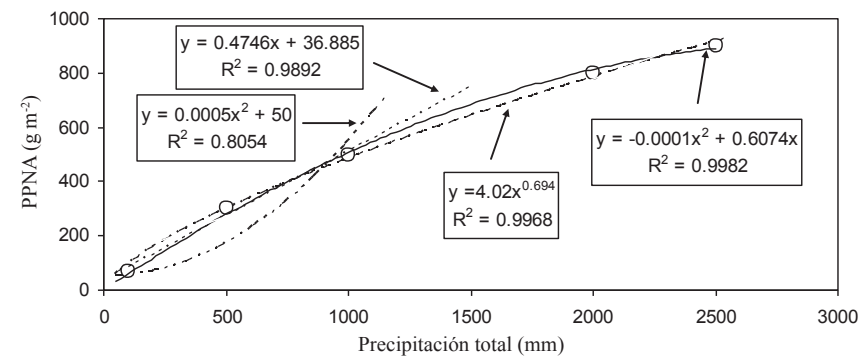

Figura 5. Ajuste de los modelos funcionales a la relación P-PPNA. probados (modelo de saturación) para P-PPNA y en la Figura 8 se muestra la relación P-EUP para este modelo.

En el apartado siguiente se discuten las relaciones funcionales analizadas, donde se usó evidencia experimental derivada de índices espectrales de vegetación, como indicadores de la PPNA o Bm. Así, es posible discriminar, como primera aproximación, la consistencia de los modelos utilizados entre P-PPNA y P-EUP, para el desarrollo de relaciones funcionales consistentes con la evidencia experimental.

\section{Modelo SESS y sus Hipótesis Intrínsecas}

Díaz-Solís et al. (2003) propusieron el modelo SESS (Simple Ecological Sustainability Simulator) para aproximar la capacidad de carga animal en el agostadero. Los desarrollos que se muestran enseguida están con base en Díaz-Solís et al. (2003), que establece que este modelo fue desarrollado para zonas áridas, con un intervalo de precipitación de 300 a $700 \mathrm{~mm}$ anuales.

En la parte de la producción primaria neta aérea anual, su estimación con base en el concepto de eficiencia del uso de la precipitación (EUP; $\mathrm{kg}$ de materia seca aérea producida por hectárea) desarrollado por Le Houreou (1984):

$P P N A=(P)(E U P)(C A)$

donde: $\mathrm{P}$ es la precipitación anual ( $\mathrm{mm}$ por año) y $\mathrm{CA}$ es la condición del agostadero (adimensional, de 0 a 1). La formulación de la relación (7) se fundamenta en el concepto de capacidad de carga utilizado por COTECOCA (1967) en su metodología de estimación de las capacidades de carga ganadera en los sitios de productividad forrajera de México. La condición del agostadero ajusta el valor potencial de producción por efectos locales (similar al concepto de COTECOCA).

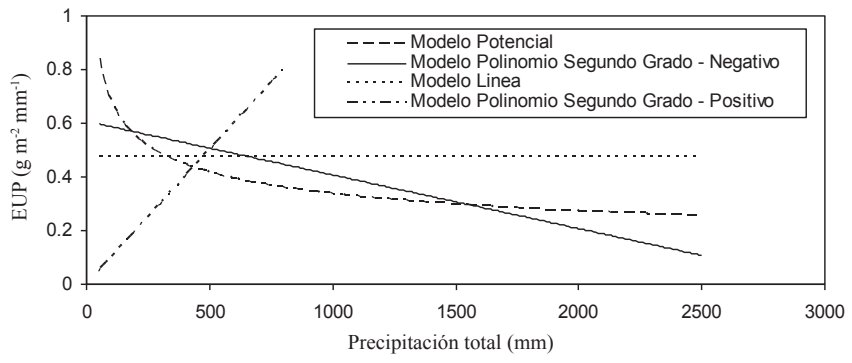

Figura 6. Relaciones precipitación $(P)$ - eficiencia en el uso de la precipitación (EUP) de los modelos ajustados en la Figura 6. 


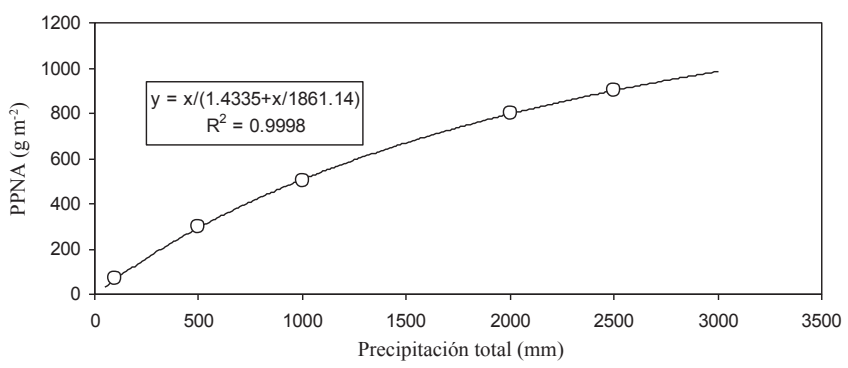

Figura 7. Ajuste del modelo de saturación a la relación P-PPNA.

La eficiencia del uso de la precipitación es estimada por medio de:

$$
E U P=C S+b_{\mathrm{p}} P
$$

donde: $b_{p}=0.0084$ y CS es un índice de condición del suelo estimado como:

$$
C S=D F+S F
$$

donde: DF es un factor que depende de la profundidad del suelo y SF es un factor de pendiente del terreno. En el Cuadro 1 se muestran los valores de SF y DF para los factores que representan.

La condición del agostadero asociada a clases está dada por los siguientes factores: condición excelente con $\mathrm{CA}=1.25$; condición buena con $\mathrm{CA}=1$; condición regular con $\mathrm{CA}=0.75$ y condición mala con $\mathrm{CA}=0.50$. De las discusiones de las secciones anteriores, el modelo SESS puede ser discutido con relación a las hipótesis intrínsecas o implícitas:

a) La relación (8) implica un polinomio de segundo grado - positivo (el valor de CS no incide en esto) entre $P$ y PPNA. Si la evidencia experimental es contraria a este modelo, entonces se requiere revisar la relación (8). No obstante lo anterior, considerando las condiciones

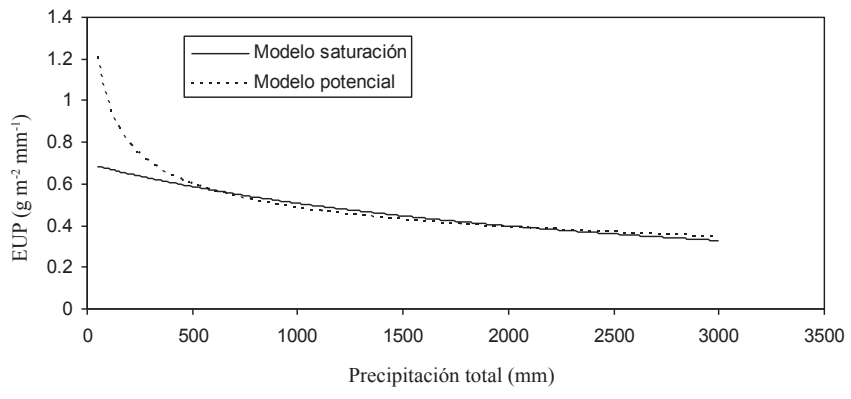

Figura 8. Relación precipitación (P) - eficiencia en el uso de la precipitación (EUP) del modelo de saturación. de aplicación del modelo SESS (300 a $700 \mathrm{~mm}$ de precipitación), es esperado que el patrón de saturación no se presente.

b) La relación (7) y (8) muestran un modelo:

$$
P P N A=(C S x C A) P+\left(C A x b_{\mathrm{p}}\right) P^{2}
$$

donde el signo asociado a $\mathrm{P}^{2}$ es positivo, implicando un crecimiento sin saturación, inconsistente con la evidencia experimental; aun para el caso de acotar $\mathrm{P}$ a valores bajos. La relación (10) muestra que para $\mathrm{P}=0$, entonces PPNA $=0$, resultando consistente en este sentido. Asimismo, la pendiente de la relación no lineal entre P y PPNA es modulada por la condición del agostadero CA, y en menor medida por la condición del suelo CS, consistente con la discusión previa de las secciones anteriores.

c) El ajuste de la condición del suelo solo considera la profundidad de éste y la pendiente del terreno y no la textura del suelo, siendo inconsistente con la evidencia experimental asociada a la hipótesis de la textura inversa; aunque es claro que la profundidad puede ser un factor que limita fuertemente el crecimiento de la vegetación.

El modelo SESS, en lo general, es consistente con la evidencia experimental, requiriendo ajustes en la relación entre $\mathrm{P}$ y la EUP, además de considerar el efecto de la textura en la condición del suelo. Otros ajustes pueden llevarse a cabo en función de la información disponible. Por ejemplo, se pueden realizar ajustes en la condición del agostadero en función a la cercanía de corrientes de agua, ambientes ribereños, para ser consistente con la metodología empleada por COTECOCA (1967). Esto puede ser hecho fácilmente usando la información disponible del INEGI en formato digital.

Cuadro 1. Valores de los factores SF y DF.

\begin{tabular}{lcc}
\hline Característica & Descripción & DF o SF \\
\hline Profundidad del suelo & $<25 \mathrm{~cm}$ & -0.5 \\
& $25-50 \mathrm{~cm}$ & 0.0 \\
& $>50 \mathrm{~cm}$ & 0.5 \\
Pendiente del terreno & $<15 \%$ & 0.5 \\
& $15-35 \%$ & 0.0 \\
& $>35 \%$ & -0.5 \\
\hline
\end{tabular}


Más adelante se discuten diversas mejoras, operativas, para incrementar la capacidad del modelo SESS (intervalo de precipitación hasta los 1500 a $2000 \mathrm{~mm}$ anuales), para caracterizar la enorme diversidad de patrones asociados a los ecosistemas, y su manejo, en México. Además del módulo de biomasa del SESS, es necesario realizar ajustes al de herbivoría con factores como la proporción de biomasa utilizable, la selectividad animal y la conversión a producto animal.

\section{Modelado de los Patrones Espacio-Temporales de los Î́ndices de Vegetación}

Considerando que la discusión del apartado anterior fue enfatizada para el caso de los ecosistemas de pastizales y matorrales, en este apartado se analizan estos ecosistemas.

Debido a las condiciones climáticas del norte de México, principalmente de baja precipitación y bajas temperaturas, la principal actividad para el sustento de sus habitantes ha sido la ganadería extensiva. La región conocida como Desierto Chihuahuense está comprendida principalmente por los estados de Nuevo León, Coahuila, Chihuahua, Zacatecas, San Luis Potosí y Durango. El mayor problema que enfrenta esta región es la pérdida de cobertura vegetal que reduce la producción animal, promueve la erosión del suelo y afecta el ciclo hidrológico (Knight, 1999¹). La principal causa ha sido la sobre utilización de la vegetación nativa por rumiantes domésticos (Ibarra et al., 19982; Olivares e Ibarra, 199933 Redmon, 19994).

El primer esfuerzo en México por conocer las condiciones de los agostaderos en esta región fue la de COTECOCA de 1968 a 1980 (COTECOCA, 1979). Sin embargo, desde estos esfuerzos hace más de 35 años, no se han realizado actualizaciones sobre el estado de estos ecosistemas y las decisiones sobre su manejo se basan en el empirismo de productores y técnicos.

Existen nuevas herramientas para dar seguimiento sistemático a diversas características de los ecosistemas con alta frecuencia en grandes superficies, pero se requiere calibrar las herramientas y los procesos con información de campo. En términos generales estas herramientas son los sensores remotos, los modelos de simulación y los sistemas de apoyo para la toma de decisiones en sistemas complejos.

\section{Sensores Remotos e Índices Espectrales de la Vegetación}

La tecnología de los sensores remotos permite el seguimiento del crecimiento y desarrollo de la vegetación, mediante la aplicación de relaciones empíricas calculadas entre las variables biofísicas de la vegetación ( $\mathrm{fv}$ o porcentaje de la cobertura aérea del follaje, IAF o índice de área foliar, biomasa o $\mathrm{Bm}$ ) y los índices de vegetación (IV) espectrales, de los cuales existe un gran número (Verstraete y Pinty, 1996; Paz et al., 2007). La mayoría de los IV están con base en el alto contraste entre la banda del rojo (R) y la del infrarrojo cercano (IRC) proveniente de la vegetación (Tucker, 1979). Los efectos atmosféricos, la geometría sol-sensor y la mezcla suelo-vegetación son factores que influyen fuertemente en los índices de vegetación. En particular, la variación en el brillo y color del suelo reviste mucha importancia para las estimaciones de variables biofísicas. Para reducir este efecto se han diseñado diferentes IV que minimizan la respuesta del índice a una misma cantidad de cubierta vegetal, variando las condiciones del suelo de fondo, tales como los índices GESAVI (Gilabert et al., 2002) y el NDVIcp (Paz et al., 2007), cuyo intervalo de validez está limitado hasta una primera fase exponencial de los patrones espectrales asociados al crecimiento de la vegetación (curvas iso-IAF). Romero et al. (2009) han generalizado el NDVIcp para el caso de las fases exponencial y lineal (hasta el IAF máximo) usando el índice IV CIMAS.

El uso de IV en la estimación de la biomasa del agostadero usando imágenes satelitales (Thoma et al., 2002; entre otros) ha sido planteado como una estrategia para tener inventarios. No obstante esto, los resultados obtenidos hasta la fecha en este tipo de ejercicios no han sido los esperados y los productores encuentran diferencias entre los estimados satelitales y su realidad a nivel de predios ganaderos (Rowley et al., 2007).

\footnotetext{
${ }^{1}$ Knight, R. W. 1999. Water conservation on grazinglands. Proceedings. Conservation and use of natural resources and marketing of beef cattle. January $27-29$. Universidad Autónoma de Nuevo León. Monterrey, Nuevo León, México.

${ }^{2}$ Ibarra, H., R. Vásquez, A. Mora, and G. Nava. 1998. Range ecosystems in northeast México and south Texas. Workshop Proceedings. Management of grazing lands in northern México and south Texas. June 25-26. Texas A\&M International University, Laredo Texas.

${ }^{3}$ Olivares, E. and H. Ibarra. 1999. Soil conservation and management on rangelands of northeast México. Proceedings. Conservation and use of natural resources and marketing of beef cattle. January 27-29. Universidad Autónoma de Nuevo León. Monterrey, Nuevo León, México.

${ }^{4}$ Redmon, L. A. 1999. Conservation of soil resources on lands used for grazing. Proceedings. Conservation and use of natural resources and marketing of beef cattle. January 27-29. Universidad Autónoma de Nuevo León. Monterrey, Nuevo León, México.
} 
El uso de sensores remotos para dar seguimiento al agostadero en México ha sido implementado en términos operativos en la implementación de seguros ganaderos paramétricos satelitales (AGROASEMEX, 2006; Paz et al., 2006). Estos seguros paramétricos satelitales utilizan el índice de vegetación NDVIcp (Paz et al., 2007), el cual fue diseñado para reducir los efectos de los suelos en la mezcla suelo-vegetación, ya que este efecto es importante en pastizales y matorrales. Los efectos atmosféricos del índice han sido minimizados (Paz et al., 2008), así como los relativos a la geometría de visión-iluminación solar (Bolaños et al., 2007). Para tener una serie temporal histórica de curvas de crecimiento de la vegetación, el seguro satelital utilizó el sensor AVHRR (Advanced Very High Resolution Sensor), el cual tiene una dimensión espacial de pixel de $1.1 \mathrm{~km} \times 1.1 \mathrm{~km}$ a nadir $\mathrm{y}$ las dimensiones aumentan con ángulos diferentes a nadir. Las curvas de crecimiento, a nivel de píxeles individuales de dimensiones estándar de $1 \mathrm{~km}$ x $1 \mathrm{~km}$ de una retícula nacional, se estima usando un modelo paramétrico ajustado a los IV observados (Paz et al., 2011).

\section{Modelos de Simulación}

Los rumiantes domésticos dependen de la vegetación natural y ésta a su vez depende del clima, del suelo y del manejo (frecuencia e intensidad de pastoreo). La temperatura y la precipitación son determinantes en el crecimiento de las plantas; Lieth (1975) llevo a cabo un análisis de la productividad primaria de los principales tipos de vegetación en el mundo y reportó para el bosque tropical lluvioso una producción primaria neta de 10 a 35 toneladas de materia seca (MS) por hectárea por año, para pastizales tropicales el rango fue de 2 a 20 y para matorrales xerófilos de 0.1 a $2.5 \mathrm{Mg} \mathrm{ha}^{-1} \mathrm{año}^{-1}$. En un estudio en los pastizales del centro de Estados Unidos, la precipitación explicó el 92\% de la variabilidad en la producción de biomasa (Lane et al., 1998).

Los pastizales naturales en el mundo se encuentran en áreas que reciben de 250 a $1000 \mathrm{~mm}$ anuales de precipitación y temperatura media anual entre $0 \mathrm{y}$ $26{ }^{\circ} \mathrm{C}$, aunque con mayor frecuencia en áreas con menos de $700 \mathrm{~mm}$ anuales (Lauenroth, 1979). De acuerdo a un estudio en 52 sitios en diferentes partes del mundo, la PPNA en pastizales con menos de $600 \mathrm{~mm}$ anuales y temperatura media anual superior a los $14{ }^{\circ} \mathrm{C}$ (condiciones relativamente semejantes a las del Norte de México) fue de 1 a $2 \mathrm{Mg} \mathrm{ha}^{-1} \mathrm{año}^{-1}$. No se encontró una relación significativa entre la temperatura media anual y la PPNA, en cambio la relación de la PPNA con la precipitación media anual fue positiva y significativa $\left(\mathrm{R}^{2}=0.51\right)$, el valor de la pendiente de la línea de regresión fue de 5; es decir, que por cada milímetro de precipitación fueron producidos $5 \mathrm{~kg}$ de MS ha ${ }^{-1}$ (Lauenroth, 1979).

Le Houreou (1984) y Le Houreou et al. (1988), usando el concepto de EUP, concluyeron que en las zonas áridas y semi áridas del mundo, el EUP es en promedio de $4 \mathrm{~kg} \mathrm{MS} \mathrm{ha-1} \mathrm{mm}^{-1} \mathrm{y}$ que el intervalo de valores es de 1.5 a 7.2. Estos autores mencionan que este valor es menor a medida que la precipitación es menor, ya que se incrementa la proporción de lluvias inapreciables.

En los últimos años se ha debatido sobre la relación entre la EUP y la precipitación. Huxman et al. (2004) concluyeron que la relación entre estas variables es negativa, pero un análisis más detallado en el altiplano árido de Mongolia (Bai et al., 2008) demostró que la EUP tiene una relación positiva con la precipitación cuando es evaluada en diferentes ambientes, pero su relación es negativa cuando se evalúa en el mismo ambiente con la variabilidad de la precipitación entre años y reportan para diferentes ambientes EUP promedio entre 2 hasta $7 \mathrm{~kg} \mathrm{MS} \mathrm{ha}{ }^{-1} \mathrm{~mm}^{-1}$ en el rango de 100 a $525 \mathrm{~mm}$ y con una relación positiva entre estas variables. Guevara et al. (2009) en el rango de precipitación de 200 a $500 \mathrm{~mm}$ anuales, consignaron valores de EUP de 3.9 a $4.8 \mathrm{~kg} \mathrm{MS} \mathrm{ha}^{-1} \mathrm{~mm}^{-1}$, en la región desértica de Argentina (Monte), lo que es consistente con los informes anteriores. Estos autores reportan valores de EUP de 3.7 a 9.4 en siembras de especies introducidas en la misma región.

Díaz-Solís et al. (2003) plantearon que esta variación se puede representar como: $\mathrm{EUP}=0.0084 * \mathrm{P}$, donde $\mathrm{P}$ es la precipitación media anual en el rango de 300 a $600 \mathrm{~mm}$, de forma que la EUP es igual a 2.52, 3.36, 4.2 y $5.04 \mathrm{~kg} \mathrm{MS} \mathrm{ha}^{-1} \mathrm{~mm}^{-1}$ en ambientes con precipitación anual de 300, 400, 500 y $600 \mathrm{~mm}$, respectivamente. Bajo este planteamiento se desarrolló SESS (Simple Ecological Sustainability Simulator), un modelo de simulación de la ganadería extensiva en el Norte de México. El modelo SESS simula la producción de forraje, su utilización y la producción animal en el sistema de producción extensivo vaca-becerro, típico del Norte de México. Está programado en STELLA ${ }^{\circledR}$ 
Research 6.0 y su objetivo es ser una herramienta simple para evaluar diferentes opciones de gestión del ganado en el sistema de producción vaca-becerro del NE de México y Sur de Texas y apoyar el diseño de políticas orientadas a este sector (Díaz-Solís et al., 2003, 2006, 2009). Este modelo ha sido validado con información del Norte de Texas con buenos resultados (Teague et al., 2008, 2009).

\section{Información Climática y Espectral}

Para este trabajo se empleó la información de la retícula nacional de índices de vegetación (IV) y climática en su escala $20 \mathrm{~km} \times 20 \mathrm{~km}$ para los pastizales y matorrales del estado de Coahuila de 1986 a 2008 y el modelo SESS.

La información de la retícula climática para cada uno de los 434 puntos (áreas o sitios) de Coahuila fue utilizada para parametrizar el modelo SESS, simulando la dinámica mensual de la biomasa de 1986 a 2008. Los valores de biomasa verde $(\mathrm{BmV})$ obtenidos fueron seleccionados de las series producidas por el modelo en cada año y sitio, dado que el índice NVIcp (Paz et al., 2007) está relacionado con esta variable (Paz, et al., 2009). Los valores producidos por SESS (simulados) se relacionaron con los valores transformados de la retícula de índices de vegetación (observados) para detectar su grado de relación, y convertir los IV a variables con mayor significado biológico y ecológico.

El modelo del crecimiento esta mostrado en la Figura 9 (AGROASEMEX, 2006; Paz et al., 2006 y 2011), que utiliza la propiedad de que el NDVIcp tiene una relación lineal con el IAF y la Bm (dada la relación lineal con el IAF), pasando por el origen ( $\mathrm{Paz}$ et al., 2007; Romero et al., 2009). En el tiempo, la evolución del NDVIcp es del tipo no lineal (Romero et al., 2009), por lo que para fines operativos este patrón fue linealizado usando una transformación inversa, Figura 9b. La base de imágenes satelitales históricas utilizadas fue de 1986 al 2008 (nivel diario del sensor AVHRR).

En los modelos de la Figura 9 la asíntota puede presentarse o no. La Figura 10 muestra un ejemplo del ajuste del modelo a los NDVIcp (multiplicado por mil) de un píxel individual, donde no se presenta una asíntota. El algoritmo de ajuste usa técnicas de reconocimiento de patrones y procesos iterativos de discriminación de valores del NDVIcp con ruidos residuales (atmósfera, degradación espectral, etc.), hasta llegar a un ajuste estadístico con una $\mathrm{R}^{2}$ mínima (0.85) (Paz et al., 2011)
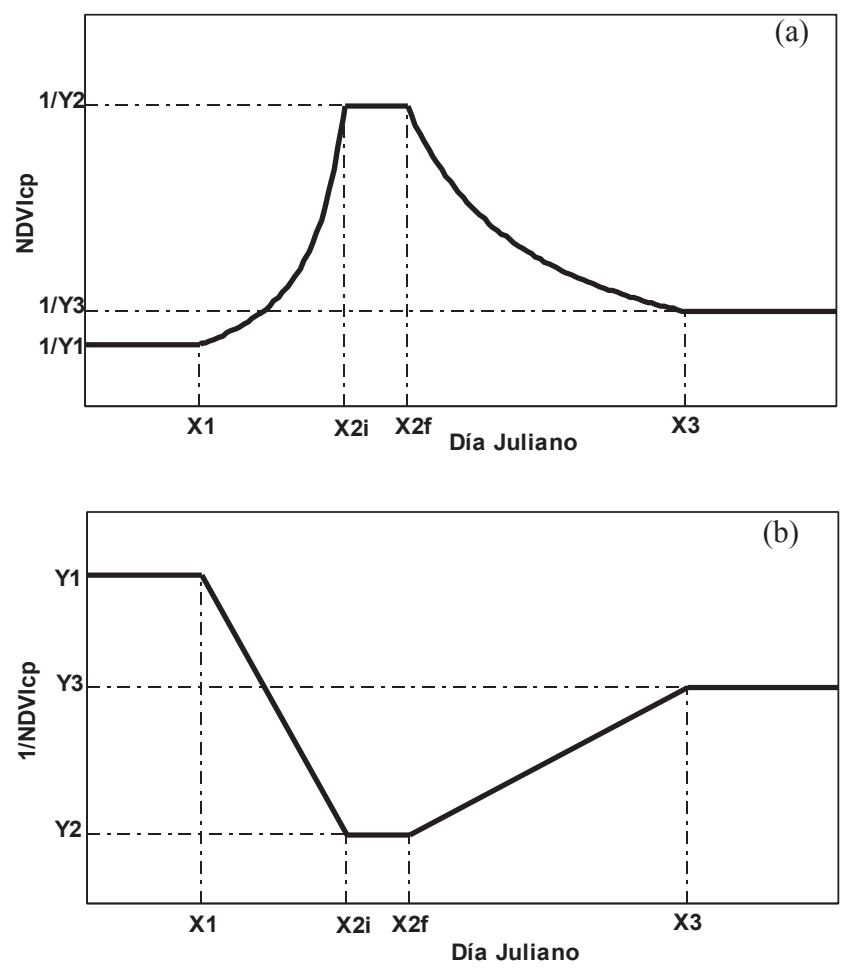

Figura 9. Patrones temporales del NDVIcp, (a) formato normal y (b) transformado.

Para analizar las relaciones entre los IV y las estimaciones de biomasa del modelo SESS se consideró que dada la relación lineal entre la biomasa y el NDVIcp (Romero et al., 2009), se consideró los recíprocos $($ NDVIcp $=1 / Y$, Figura 9a) de los IV usados en el seguro ganadero paramétrico satelital. Así, solo se consideró el índice NDVIcp máximo (1/Y2), dado que la acumulación de biomasa total (suma de la etapa creciente y decreciente de la Figura 9a) muestra una alta correlación con el máximo (Paz, 2008).

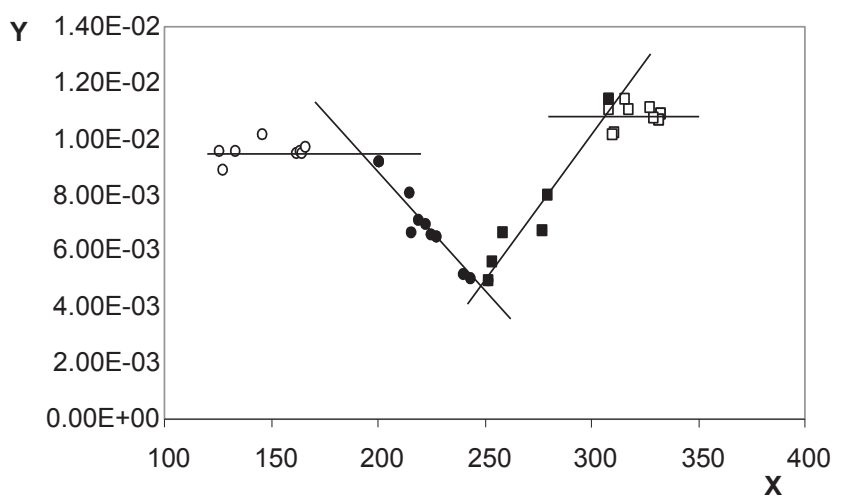

Figura 10. Ejemplo de parametrización del modelo temporal del NDVIcp ( $Y=1 /$ NDVI $_{\mathrm{cp}}, \mathrm{X}=$ tiempo en días julianos). 
Para realizar un análisis en términos de la precipitación asociada a un año en particular, los IV fueron estandarizados al dividirlos por la precipitación acumulada anual. En el caso de las precipitaciones, se utilizó una retícula nacional de datos de precipitación, y temperaturas, diarios de 1979-2008 proporcionados por AGROASEMEX, S.A., los cuales fueron muestreados nuevamente por el Colegio de Postgraduados a una retícula de $20 \mathrm{~km} \times 20 \mathrm{~km}$, donde se ubicaron las estimaciones de curvas de crecimiento asociadas al NDVIcp de 1986 al 2008. Las estimaciones de AGROASEMEX se hicieron con el uso de la información disponible de las estaciones meteorológicas nacionales disponibles, después de un proceso estricto de control de calidad y certificación internacional. Además de un proceso de asimilación de las estimaciones de precipitación y temperatura de los modelos de circulación atmosférica global, de tal forma que esta base de datos tuviera congruencia local y global.

\section{RESULTADOS Y DISCUSIÓN}

\section{Patrones Espectrales de la Vegetación en Coahuila y su Modelado}

Con anterioridad se han utilizado índices de vegetación para analizar la relación P-Bm y P-PPNA (Prince et al., 1998; Paruelo et al., 1999; entre otros) por medio del índice NDVI [= (IRC-R)/(IRC+R)], el cual está limitado debido a sus hipótesis intrínsecas que son inconsistentes con los patrones temporales del crecimiento de la vegetación (Paz et al., 2007). Para evitar el problema de la utilización de índices de vegetación no directamente asociados al crecimiento de la vegetación, en lo siguiente se utiliza el índice NDVIcp.

En el caso de los patrones entre el NDVIcp (1000/Y2) y P $(\mathrm{mm})$ de los 7910 píxeles de $20 \mathrm{~km} \times 20 \mathrm{~km}$ (píxeles puros y de mezclas de pastizales, matorrales y bosques; definiciones del INEGI), la Figura 11 muestra los patrones observados para todo el estado de Coahuila.

Se observa en la Figura 11 que existe una constante aditiva en el NDVIcp que puede ser atribuida a la radiancia de trayectoria atmosférica (Paz et al., 2008). Esta constante aditiva, más una multiplicativa, no modifica los argumentos siguientes ya que los patrones generales observados permanecen por una transformación afín (lineal).

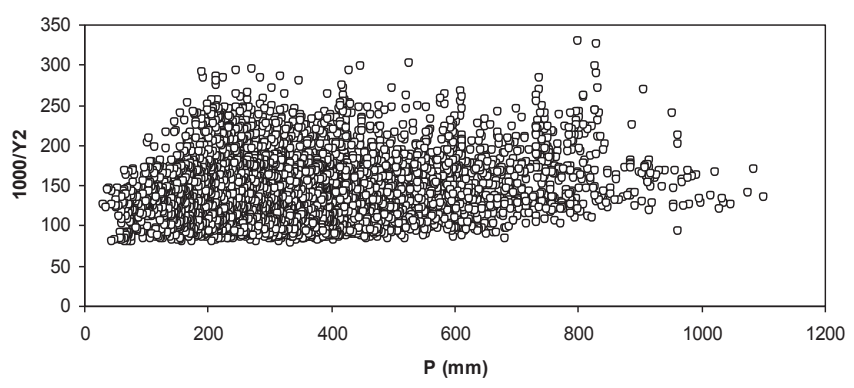

Figura 11. Patrones de la relación $\mathrm{P}-(\mathbf{1 0 0 0} / \mathrm{Y} 2=1000 x$ NDVIcp $)$ para Coahuila. $\mathbf{P}=$ precipitación.

Tal como se discutió anteriormente, y con el uso de un enfoque similar al de Prince et al. (1998), se puede analizar la relación entre la EUP (= NDVIcp/P) y P (Figura 12). Prince y colaboradores usaron una serie temporal de solo 9 años y como indicador de la PPNA utilizaron el acumulado del NDVI (que es una variable acumulada por definición), que produce correlaciones espurias (Malet et al., 1997).

Del patrón potencial, o aproximación semejante, puede concluirse que el modelo lineal usado en el SESS es inconsistente con la evidencia experimental. El patrón de la Figura 12 apoya el modelo potencial y de saturación.

Al considerar que el exponente de la relación entre P y EUP es cercano a 1 (Figura 12), podemos transformar el eje de P y usar su inverso (1000/P), para linealizar los patrones observados (Figura 13).

Ahora bien, para analizar la posibilidad de predicción de la biomasa aérea verde $(\mathrm{BmV})$, la Figura 14 muestra la misma relación que la Figura 13, al sustituir $(1000 / \mathrm{P})$ por $(1000 / \mathrm{BmV})$ simulada con el modelo SESS.

En las Figuras 13 y 14 se forzaron las regresiones lineales a pasar por el origen, ya que la constante aditiva no es significativamente diferente de cero.

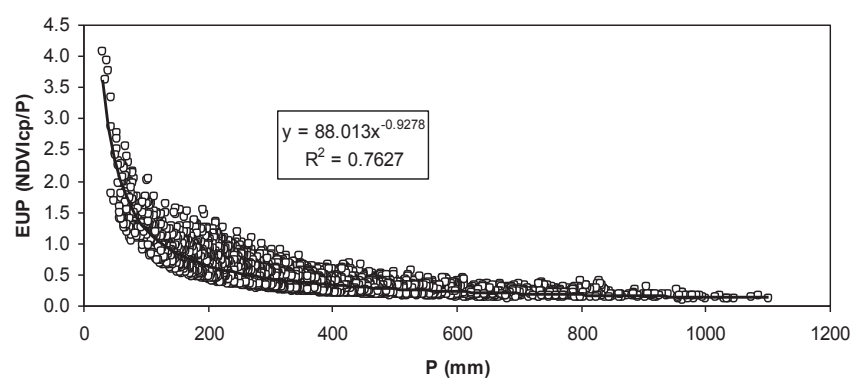

Figura 12. Relación entre P y EUP para el estado de Coahuila. $\mathbf{E U P}=$ eficiencia en el uso de la precipitación; $\mathbf{P}=$ precipitación. 


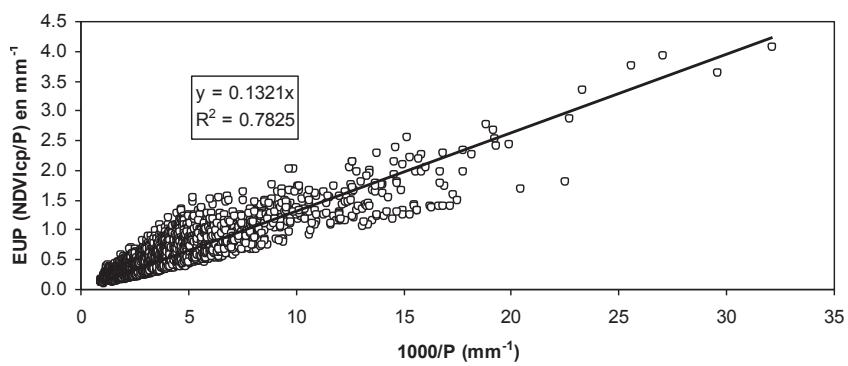

Figura 13. Relación entre el reciproco de precipitación $(P)$ y la eficiencia en el uso de la precipitación (EUP).

De la Figura 14, y las discusiones previas, se puede plantear el ajuste de un modelo lineal (no lineal transformado a lineal) dependiente (pendiente $\mathrm{B}$ de la relación 1000/BmV y EUP) de las condiciones del agostadero y del tipo de ecosistema (Figura 15).

Para diferenciar las condiciones del agostadero, se puede plantear la hipótesis de que sus respuestas funcionales (curvas de crecimiento y picos del crecimiento) se pueden caracterizar por la relación entre la pendiente $\mathrm{B}(1 / \mathrm{NDV} c \mathrm{p})$ (Figura 16).

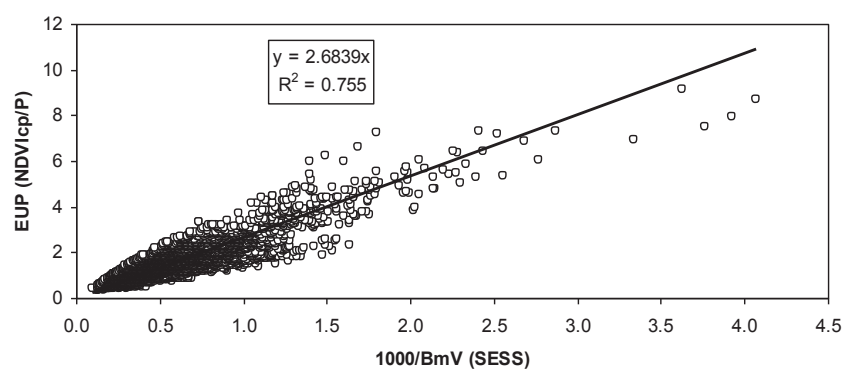

Figura 14. Relación entre el reciproco de BmV y la EUP.

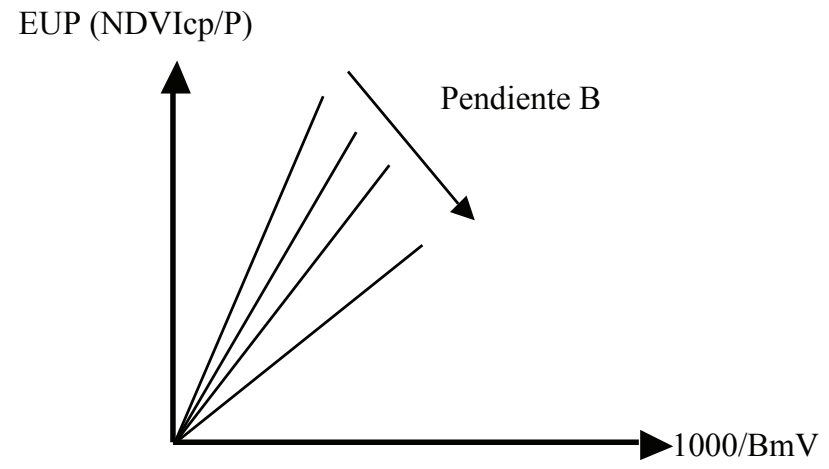

Figura 15. Modelo entre $(1000 / \mathrm{BmV})$ y EUP para diferentes condiciones del agostadero.

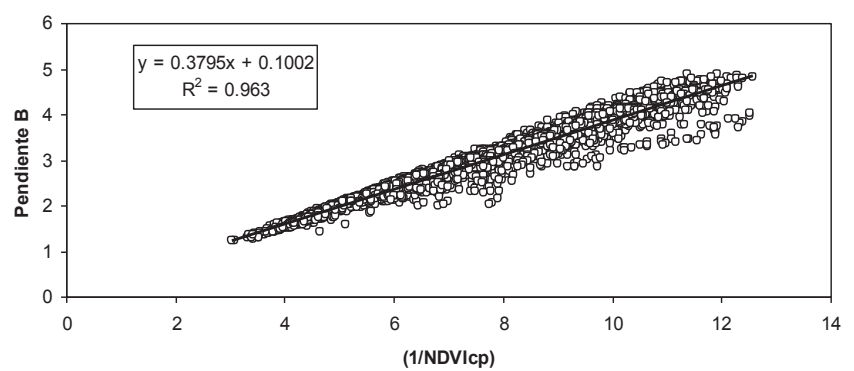

Figura 16. Relación entre (1/NDVIcp) y la pendiente B.

Usando el modelo, de aproximación de la relación de la Figura 16, $\mathrm{B}=0.346(1 / \mathrm{NDVIcp})$, la Figura 17 muestra las estimaciones de $(1000 / \mathrm{BmV})$ con relación a las del modelo SESS. En la Figura 17 se muestra en una línea discontinua un patrón diferente producto de la inconsistencia de la hipótesis del modelo SESS de usar EUP $=\mathrm{a}+\mathrm{bP}$; aunque dado que este modelo fue desarrollado para el intervalo entre 300 y $700 \mathrm{~mm}$ anuales de precipitación (sin que se presente el patrón de saturación), el ajuste de la versión actual del modelo SESS mostrada en la Figura 17 resulta un buen ajuste.

Los resultados mostrados permiten establecer una aproximación simple de modelar la relación entre el índice espectral NDVIcp (relacionado linealmente con la $\mathrm{Bm}$ ) y la precipitación total $\mathrm{P}$, que es consistente con el uso de modelos relativamente simples de procesos ecológicos y de manejo.

El ejercicio realizado permite el desarrollo de una aproximación de modelación hibrida (sensores remotos y modelos biofísicos) que puede desarrollarse para considerar diferentes condiciones asociadas a los ecosistemas terrestres, en lo particular al manejo de pastizales tropicales y bosques/selvas. En ambos casos es necesario el desarrollo de una estrategia más global para la modelación de la relación clima-Bm o clima-PPNA, de acuerdo con la formulación de las relaciones (2).

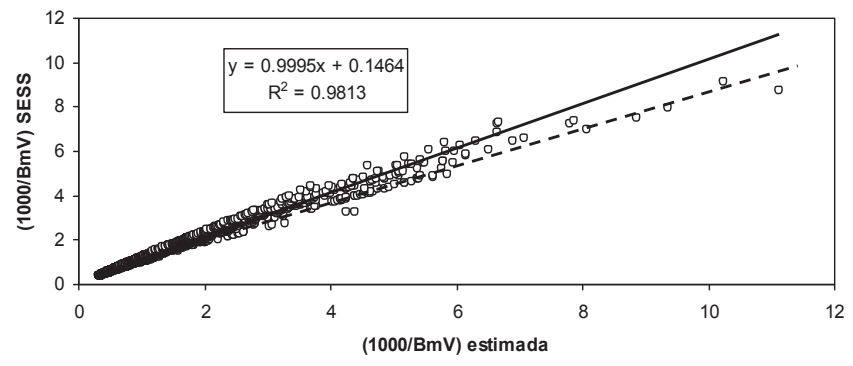

Figura 17. Relación entre las estimaciones de $(1000 / \mathrm{Bm})$ comparadas con las simuladas por el modelo SESS. 


\section{CONCLUSIONES Y NUEVOS DESARROLLOS}

- Además de las mejorías planteadas para el modelo SESS, una estrategia para caracterizar (diferenciar) ecosistemas terrestres y su condición (degradación) consiste en el desarrollo de algunos planteamientos de extensión. El modelo SESS resulta adecuado en aplicaciones donde la eficiencia en el uso de la precipitación considerada sea válida, requiriendo su modificación para el caso general, tal como fue discutido en este trabajo; aunque para fines prácticos los resultados del modelo SESS convencional muestran resultados razonables y similares a los obtenidos con las modificaciones propuestas.

- Usando la retícula climática de AGROASEMEX (P, Tmin y Tmax) se puede usar el intervalo de temperatura (Tmax - Tmin) para obtener una primera aproximación de la radiación solar (Bristow y Campbell, 1984; Goodin et al., 1999) o usar estimaciones de sensores remotos (Garatuza-Payan et al., 2001) y usar las relaciones (2) para extender la aproximación del enfoque de la EUP y considerar la situación del crecimiento de la vegetación bajo condiciones de limitaciones de energía (bosques/ selvas y pastizales cultivados), de tal forma que pueda usarse un modelo integrado de los principales factores (precipitación y radiación; además de las variables asociadas a los balances hídricos del suelo) que inciden en el crecimiento de la vegetación en los ecosistemas terrestres en México. Los efectos nutrimentales en el crecimiento de la vegetación pueden ser considerados en la condición del suelo usando las bases de datos de suelos y propiedades físicas/químicas disponibles.

- Resulta importante desarrollar un enfoque que considere en forma explícita que los índices espectrales de la vegetación representan la biomasa total (acumulados anuales para el caso de matorrales y bosques/selvas) y no solo los incrementos anuales (PPNA). Este enfoque permitiría construir curvas de crecimiento anual de matorrales y bosques/ selvas al considerar acumulados multi-anuales de la precipitación (y radiación), permitiendo fechar información de campo con muestreos uni-temporales. Lo anterior es importante para analizar la información asociada a la biomasa aérea, producto de estimaciones alométricas, disponible de los inventarios forestales y de suelos de la CONAFOR.
- En la perspectiva de caracterizar, primera aproximación, los ecosistemas terrestres de México, se puede usar el planteamiento que la EUP (y la eficiencia de uso de la radiación) define patrones específicos en relación al crecimiento (PPNA), para su caracterización en términos adecuados y etiquetarlos como clases genéricas de vegetación. Bajo el concepto de variación de la EUP (y para la radiación) para los ecosistemas pastizales, matorrales y bosques/selvas, bajo diferentes condiciones del "agostadero" (generalizado para el caso de bosques/selvas), se puede analizar los cambios en estas eficiencias como mediciones indirectas de la degradación de estos ecosistemas. Esto se puede usar con la malla climática de AGROASEMEX y las estimaciones de biomasa de los inventarios forestales de la CONAFOR y SARH (de Jong et al., 2010), usando como estrategia el análisis de patrones climaPPNA (y su acumulado estimado de los inventarios) para condiciones fijas del clima (por ejemplo, misma precipitación). Esta estrategia permite una primera aproximación a la definición del concepto de degradación de un ecosistema, además de diferenciar razonablemente los tipos de ecosistemas, definidos por el INEGI, y así caracterizar la incertidumbre asociada al etiquetado (dependiente de escala) de clases de vegetación.

\section{LITERATURA CITADA}

AGROASEMEX, S.A. 2006. La experiencia mexicana en el desarrollo y operación de seguros paramétricos orientados a la agricultura. AGROASEMEX, S.A. Querétaro, México. ISBN 968-9098-00-4.

Arnold, J. G., M. A. Weltz, E. E. Alberts, and D. C. Flanagan. 1995. Plant growth component. Chapter 8. pp. 8.1-8.41. In: D. C. Flanagan and M. A. Nearing (eds.). USDA-Water Erosion Prediction Project (WEPP). Hillslope profile and watershed model documentation. NSERL Report No. 10. USDA-ARS National Soil Erosion Research Laboratory. West Lafayette, IN, USA.

Austin, A. T. 2002. Differential effects of precipitation and decomposition along a rainfall gradient in Hawaii. Ecology 83: 328-338. doi: 10.2307/2680017.

Bai, Y., J. Wu, Q. Xing, Q. Pan, J. Huang, D. Yang, and X. Han. 2008. Primary production and rain use efficiency across a precipitation gradient on the Mongolia plateau. Ecology 89: 2140-2153. doi: 101890/07-0992.1.

Blanco, L. J., C. A. Ferrando, R. E. Quiroga, E. L. Orionte, D. J. Recalde, F. N. Biurrun, and G. D. Berone. 2005. Relación entre producción forrajera y precipitación en comunidades vegetales autóctonas de Los Llanos de La Rioja. Rev. Argentina Producc. Anim. 25: 200-201. 
Bolaños, M., F. Paz, E. Palacios, E. Mejía y A. Huete. 2007. Modelación de los efectos de la geometría sol-sensor en la reflectancia de la vegetación. Agrociencia 41: 527-537.

Bristow, K. and G. S. Campbell. 1984. On the relationship between incoming solar radiation and daily maximum and minimum temperature. Agric. For. Meteorol. 31: 159-166. doi: 10.1016/0168-1923(84)90017-0.

Christie, E. K. 1981. Biomass and nutrient dynamics in a C4 semiarid Australian grassland community. J. Appl. Ecol. 18: 907918. doi: $10.2307 / 2402381$.

COTECOCA (Comisión Técnico Consultiva de Coeficientes de Agostadero). 1967. Metodología para determinar tipos vegetativos, sitios y productividad de sitios. Publicación No. 8. México, D. F.

COTECOCA (Comisión Técnico Consultiva de Coeficientes de Agostadero). 1979. Coahuila. Tipos de vegetación, sitios de productividad forrajera y coeficientes de agostadero. Secretaria de Recursos Hidráulicos. Comisión Técnico Consultiva para la Determinación Regional de los Coeficientes de Agostadero. México.

de Jong, B., C. Anaya, O. Masera, M. Olguín, F. Paz, J. Etchevers, R. Martínez, G. Guerrero, and C. Balbontín. 2010. Greenhouse gas emissions between 1993 and 2002 from land-use change and forestry in Mexico. For. Ecol. Manage. 260: 1689-1701. doi: 10.1016/j.foreco.2010.08.011.

Díaz-Solís, H., M. M. Kothmann, W. T. Hamilton, and W. E. Grant. 2003. A simple ecological sustainability simulator (SESS) for stocking rate management on semi-arid grazinglands. Agric. Syst. 76: 655-680. doi: 10.1016/S0308-521X(01)00115-9.

Díaz-Solís, H., W. E. Grant, M. M. Kothmann, W. R. Teague, and J. A. Díaz-García. 2009. Adaptive management of stocking rates to reduce effects of drought on cow calf production systems in semi-arid rangelands. Agric. Syst. 100: 43-50. doi: 10.1016/j. agsy.2008.12.007.

Díaz-Solís, H., M. M. Kothmann, W. E. Grant, and R. De Luna-Villarreal. 2006. Application of a simple ecological sustainability simulator (SESS) as a management tool in the semi-arid rangelands of northeastern Mexico. Agric. Syst. 88: 514-527. doi: /10.1016/j.agsy.2005.07.008.

Donnelly, J. R., A. D. Moore, and M. Freer. 1997. GRAZPLAN: Decision support systems for Australian grazing enterprises I. Overview of the GRAZPLAN project, and a description of the MetAccess and Lamb Alive DSS. Agric. Syst. 54: 57-76.

Doorenbos, J. and A. Kassam. 1979. Yield response to water. FAO Irrigation and Drainage Paper 33. FAO, Rome.

Epstein, H. E., W. K. Lauenroth, and I. C. Burke. 1997. Effects of temperature and soil texture on ANPP in the U.S. great plains. Ecology 78: 2628-2631.

Fang, J., S. Piao, L. Zhou, J. He, F. Wei, R.B. Myneni, C. J. Tucker, and K. Tan. 2005. Precipitation patterns alter growth of temperate vegetation. Geophys. Res. Lett. Vol. 32. doi:10.1029/2005GL024231.

Freer, M., A. D. Moore, and J. R. Donnelly. 1997. GRAZPLAN: Decision support systems for Australian grazing enterprises II. The animal biology model for feed intake, production and reproduction and the GrazFeed DSS. Agric. Syst. 54: 77-126. doi: 10.1016/S0308-521X(96)00045-5.
Garatuza-Payan, J., R. T. Pinker, W. J. Shuttleworth, and C. J. Watts. 2001. Solar radiation and evapotranspiration in northern Mexico estimated from remotely sensed measurements of cloudiness. Hydrol. Sci. J. 46: 465-478. doi: 10.1080/02626660109492839.

George, M. R., W. A. Williams, N. K. McDougald, W. J. Clawson, and A. H. Murphy. 1989. Predicting peak standing crop on annual range using weather variables. J. Range Manage. 42: 508-513.

Gilabert, M. A., J. González-Piqueras, F. J. García-Haro, and J. Meliá. 2002. A generalized soil-adjusted vegetation index. Remote Sens. Environ. 82: 303-310. doi: 10.1016/S00344257(02)00048-2.

Gómez, I. A. y G. C. Gallopín. 1991. Estimación de la productividad primaria neta de ecosistemas terrestres del mundo en relación a factores ambientales. Ecol. Aust. 1: 24-40.

Goodin, D. G., J. M. S. Hutchinson, R. L. Vanderlip, and M. C. Knapp. 1999. Estimating solar irradiance for crop modeling using daily air temperature data. Agron. J. 91: 845-851. doi: 10.2134/agronj1999.915845x.

Goudriaan, J. and H. H. Van Laar. 1994. Modelling potential crop growth processes. Textbook with exercises. Kluwer Academic Publishers. Dordrecht, Netherlands. ISBN: 978-0-79233220-6.

Guevara, J. C., E. G. Grünwaldt, O. R. Estevez, A. J. Bisigato, L. J. Blanco, F. N. Biurrun, C. A. Ferrando, C. C. Chirino, E. Morici, B. Fernández, L. I. Allegretti, and C. B. Passera. 2009. Range and livestock production in the Monte Desert, Argentina. J. Arid Environm. 73: 228-237. doi: 10.1016/j. jaridenv.2008.02.001.

Huxman, T. E., M. D. Smith, P. A. Fay, A. K. Knapp, M. R. Shaw, M. E. Loik, S. D Smith, D. T. Tissue, J. C. Zak, J. F. Weltzin, W. T. Pockman, O. E. Sala, B. M. Haddad, J. Harte, G. W. Koch, S. Schwinning, E. E. Small, and D. G. Williams. 2004. Convergence across biomes to a common rain-use efficiency. Nature 429: 651-654. doi: 10.1038/nature02561.

Knapp, A. K. and M. D. Smith. 2001. Variation among biomes in temporal dynamics of aboveground primary production. Science 291: 481-484. doi: 10.1126/science.291.5503.481.

Knapp, A. K., C. Beier, D. D. Briske, A. T. Classen, Y. Luo, M. Reichstein, M. D. Smith, S. D. Smith, J. E. Bell, P. A. Fay, J. L. Heisler, S. W. Leavitt, R. Sherry, B. Smith, and E. Weng. 2008a. Consequences of more extreme precipitation regimes for terrestrial ecosystems. BioScience 58: 811-821. doi: 10.16417/B580908.

Knapp, A. K., J. M. Briggs, S. L. Collins, S. R. Archers, M. S. BretHarte, B. E. Ewers, D. P. Peters, D. R. Young, G. R. Shavel, E. Pendall, and M. B. Cleary. 2008b. Shrub encroachment in North American grasslands: Shifts in growth form dominance rapidly alters control of ecosystem carbon inputs. Global Change Biol. 14: 615-623. doi: 10.1111/j.1365-2486.2007.01512.x.

Kletter, A. Y., J. von Hardenberg, E. Merou, and A. Provenzale. 2009. Patterned vegetation and rainfall intermittency. J. Theor. Biol. 256: 574-583. doi: 10.1016/j.jtbi.2008.10.020.

Lane, D. R., D. P. Coffin, and W. K. Lauenroth. 1998. Effects of soil texture and precipitation on above-ground net primary productivity and vegetation structure across the Central Grassland region of the United States. J. Veget. Sci. 9: 239-250. doi: $10.2307 / 3237123$. 
Lauenroth, W. K. 1979. Grassland primary production: North American grasslands in perspective. In: N. R. French (ed.). Perspectives in grassland ecology. Ecological studies (Analysis and Synthesis), vol. 32. Springer-Verlag. New York, NY, USA.

Lauenroth, W. K. and O. E. Sala. 1992. Long-term forage production of North American shortgrass steppe. Ecol. Appl. 2: 397-403.

Le Houerou, H. N. 1984. Rain use efficiency: A unifying concept in arid-land ecology. J. Arid Environ. 7: 213-247.

Le Houerou, H. N. 1989. The grazing land ecosystems of the African Sahel. Springer-Verlag. Berlin. ISBN 13: 978-3642744594.

Le Houerou, H. N. 2006. Environmental constraints and limits to livestock husbandry in arid lands. Secheresse 17: 10-18.

Le Houerou, H. N. and C. H. Hoste. 1977. Rangeland production and annual rainfall relations in the Mediterranean basin and in the African Sahelo-Sudanian zone. J. Range Manage. 30: 181-189.

Le Houerou, H. N., R. L. Bringham, and W. Skerbek 1988. Relationship between the variability of primary production and the variability of annual precipitation in world arid lands. J. Arid Environ. 15: 1-18.

Lieth, H. 1975. Modeling the primary productivity of the worl. pp. 237-264. In: H. Lieth and R. H. Whittaker (eds.). Primary productiviy of the biosphere. Springer-Verlag. New York, NY, USA.

Malet, P., F. Pécaut, and C. Bruchou. 1997. Beware of using cumulated variables in growth and development models. Agric. For. Meteorol. 88: 137-143. doi: 10.106/S01681923(97)00042-7.

Noy-Meir, I. 1973. Desert ecosystems: Environment and producers. Annu. Rev. Ecol. Syst. 4: 25-51.

NRCS (Natural Resources Conservation Service). 1997. National Range and Pasture Handbook. Natural Resources Conservation Service, United States Department of Agriculture. Washington, DC, USA.

Paruelo, J. M., W. K. Lauenroth, I. C. Burke, and O. E. Sala. 1999. Grassland precipitation-use efficiency varies across a resource gradient. Ecosystems 2: 64-68. doi: 10.1007/s100219900058.

Paz, F., E. Palacios, M. Bolaños, A. Cano, A. Zarco, F. Pascual, L. A. Palacios y M. Martínez. 2006. Design of a country scale livestock insurance in grasslands using AVHRR sensor. pp. 683-685. In: J. A. Sobrino (ed.). Second Recent Advances in Quantitative Remote Sensing. Universitat de Valencia. Valencia, Spain.

Paz, F., E. Palacios, M. Bolaños, L. A. Palacios, M. Martínez, E. Mejía y A. Huete. 2007. Diseño de un índice espectral de la vegetación: NDVIcp. Agrociencia 41: 539-554.

Paz, F., M. Bolaños, E. Palacios, L. A. Palacios, M. Martinez y A. Huete. 2008. Optimización del índice espectral de la vegetación NDVIcp. Agrociencia 42: 925-937.

Paz, F., A. Zarco, A. Cano, M. A. Bolaños y M. Odi. 2009. Un método simple para estimar las propiedades geométricas y ópticas de las hojas de la vegetación. Agrociencia 43: 417-425.

Paz, F., M. A. Bolaños, L. A. Palacios, F. Pascual, E. Medrano y F. Ibarra. 2011. Desarrollo de curvas espectrales del crecimiento anual de la vegetación, usando sensores remotos. Rev. Mex. Cienc. Pecu. 2: 445-464.

Prince, S. D., E. B. de Colstoun, and L. L. Kravitz. 1998. Evidence from rain-use efficiencies does not indicate extensive Sahelian desertification. Global Change Biol. 4: 359-374. doi: 10.1046/j.1365-2486.1998.00158.x.
Romero, E, F. Paz, E. Palacios, M. Bolaños, R. Valdez y A. Aldrete. 2009. Diseño de un índice espectral de la vegetación desde una perspectiva conjunta de los patrones exponenciales y lineales del crecimiento. Agrociencia 43: 291-307.

Rowley, R. J., K. P. Price, and J. H. Kastens. 2007. Remote sensing and the rancher: Linking rancher perception and remote sensing. Rangeland Ecol. Manage. 60: 359-368. doi: 10.2111/1551-5028(2007)60[359:RSATRL]2.0.CO;2.

Rosenzweig, M. L. 1968. Net primary productivity of terrestrial communities: prediction from climatological data. The American Naturalist 102: 67-74.

Sala, O. E., W. J. Parton, L. A. Joyce, and W. K. Lauenroth. 1988. Primary production of the central grassland region of the United States. Ecology 69: 40-45. doi: 10.2307/1943158.

Schuur, E. A. G. 2003. Productivity and global climate revisited: the sensitivity of tropical forest growth to precipitation. Ecology 84: $1165-1170$.

Stockle, C. O., M. Donatelli, and R. Nelson. 2003. CropSyst, a cropping systems simulation model. Eur. J. Agron. 18: 289-307. doi: 10.1016/S1161-0301(02)00109-0.

Stuth, J., D. Schmitt, R. C. Rowan, J. Angerer, and K. Zander. 2003. PHYGROW (Phytomass Growth Simulator). User's guide and technical documentation. Ranching systems group. Department of Rangeland Ecology and Management, Texas A\&M University. College Station, TX, USA.

Teague, W. R., W. E. Grant, U. P. Kreuter, H. Diaz-Solís, S. Dube, M. M. Kothmann, W. E. Pinchak, and R. J. Ansley. 2008. An ecological economic simulation model for assessing fire and grazing management effects on mesquite rangelands in Texas. Ecol. Econ. 64: 612-625. doi: 10.1016/j.ecolecon.2007.04.010.

Teague, W. R, U. P. Kreuter, W. E. Grant, H. Diaz-Solís, and M. M. Kothmann. 2009. Economic implications of maintaining rangeland ecosystem health in a semi-arid savanna. Ecol. Econ. 68: 1417-1429. doi: 10.1016/j.ecolecon.2008.10.014.

Thoma, D. P., D. W. Bailey, D. S. Long, G. A. Nielsen, M. P. Henry, M. C. Breneman, and C. Montagne. 2002. Short-term monitoring of rangeland forage conditions with AVHRR imagery. J. Range Manage. 55: 383-389.

Tucker, C. J. 1979. Red and photographic infrared linear combinations for monitoring vegetation. Remote Sens. Environ. 8: 127-150. doi: 10.1016/0034-4257(79)90013-0.

Verón, S. R., M. Oesterheld, and J. M. Paruelo. 2005. Production as a function of resource availability: Slopes and efficiencies are different. J. Veget. Sci. 16: 351-354.

Varnamkhasti, A. S., D. G. Milchunas, W. K. Lauenroth, and H. Goetz. 1995. Production and rain use efficiency in short-grass steppe: Grazing history, defoliation and water resource. J. Veget. Sci. 6: 787-796. doi: 10.2307/3236392.

Verstraete, M. M. and B. Pinty. 1996. Designing optical spectral indexes for remote sensing applications. IEEE Trans. Geosci. Remote Sens. 34: 1254-1265.

Weltzin, J. F., M. E. Loik, S. Schwinning, D. G. Williams, P. A. Fay, B. M. Haddad, J. Harte, T. E. Huxman, A. K. Knapp, G. Lin, W. T. Pockman, R. M. Shaw, E. E. Small, M. D. Smith, S. D. Smith, D. T. Tissue, and J. C. Zak. 2003. Assessing the response of terrestrial ecosystems to potential changes in precipitation. BioScience 53: 941-952. 
Webb, W. L., W. K. Lauenroth, S. T. Szarek, and R. S. Kinerson. 1983. Primary production and abiotic controls in forests, grasslands, and desert ecosystems in the United States. Ecology 64: 134-151. doi: 10-2307/1937336.

Wight, J. R. and J. W. Skiles. 1987. SPUR: Simulation of production and utilization of rangelands. Documentation and user guide. ARS 63. U.S. Department of Agriculture, ARS. Washington, DC, USA.
Wylie, B. K., R. D. Pieper, and G. M. Southward. 1992. Estimating herbage standing crop from rainfall data in Niger. J. Range Manage. 42: 277-284.

Yahdjian, L. and O. E. Sala. 2006. Vegetation structure constrain primary production response to water availability in the Patagonian steppe. Ecology 87: 952-962. doi: 10.1890/0012-9658(2006)87[952:VSCPPR]2.0.CO;2. 